\title{
Depression scores change significantly after omalizumab treatment in patients with chronic spontaneous urticaria
}

\author{
Pelin Kuteyla Can, ${ }^{1}$ Piril Etikan, ${ }^{2}$ Ece Nur Degirmentepe, ${ }^{2}$ Emek Kocaturk ${ }^{3}$
}

\begin{abstract}
Background: Chronic spontaneous urticaria (CSU) is frequently associated with psychiatric comorbidities.
\end{abstract}

Objective: We aimed to determine if depressive symptoms were present in CSU patients who received omalizumab and if depression scores got better with omalizumab treatment and whether the presence of depressive symptoms impaired treatment responses.

Methods: CSU patients who received at least three injections of omalizumab were included in the study. Changes in Urticaria Activity Score (UAS), Chronic Urticaria Quality of Life Questionnaire (CU-Q ${ }^{2} \mathrm{oL}$ ), Beck Depression Inventory (Beck-D) and Urticaria Control Test (UCT) scores were compared before and after treatment.

Results: From 49 patients, 20 (40.8\%) had depressive symptoms at baseline. After treatment, UAS7, CU-Q²oL, Beck-D scores decreased and UCT-scores increased significantly $(p<0.001$, for all). UCT scores were lower at baseline and at $3^{\text {rd }}$ month following treatment in patients with depressive symptoms compared to patients without (baseline median (interquartile range-IQR) 2.5 (1-5) vs 5 (2.5-6.5); $p=0.04$ and $3^{\text {rd }}$ month 12 (9-13) vs $14(12-16) ; p=0.006$, respectively). Omalizumab non-responders had higher baseline Beck-D-scores [18.5 (15.2-22) vs 12 (6-22.5); $p=0.031$ ]. The number of omalizumab non-responders were significantly higher among patients with depressive symptoms compared to patients without. ( $40 \%$ vs $13.8 \% ; p=0.048)$. Only 6 patients scored as having depressive symptoms after treatment; of these 6 patients only one was an omalizumab responder.

Conclusion: Omalizumab not only provides symptom control for urticaria but also improves psychological conditions of the patients. Coexistent psychiatric comorbidities should be taken into account in CSU patients since these conditions might impair treatment response.

Key words: biomarker, depression, omalizumab, psychiatric comorbidity, urticaria

\section{From:}

Medicalpark Maltepe Hospital; Dermatology and Venerology, Istanbul, Turkey

Okmeydani Training and Research Hospital; Dermatology and Venerology, Istanbul, Turkey

3 Koç University School of Medicine; Department of Dermatology, Istanbul, Turkey

\section{Corresponding author:}

Emek Kocaturk

Topkapı, Koç Üniversitesi Hastanesi, Davutpaşa Cd.

No. 4, 34010 Zeytinburnu, İstanbul

E-mail: ekocaturk@ku.edu.tr

\section{Abbrevations:}

ASST autolog serum skin test

Beck-D Beck-depression inventory

CINDU chronic inducible urticarias

CSU chronic spontanous urticaria

CU chronic urticaria

CU-Q ${ }^{2} \mathrm{oL}$ chronic urticaria quality of life

\author{
Abbrevations (Continued): \\ DLQI dermatology life quality index \\ IQR interquartile range \\ OMA omalizumab \\ QOL quality of life \\ UAS Urticaria activity score \\ UCT Urticaria control test \\ WHO-5 World Health Organization Well-being Index
}

\section{Introduction}

Chronic spontaneous urticaria (CSU) is characterized by recurrent episodes of wheals (hives) and/or angioedema for at least 6 weeks, lesions are itchy, and angioedema may present with burning and pain sensation. CSU is a long lasting disease, and all these symptoms have a substantial impact on patients' quality of life (QoL) and may lead to psychiatric consequences. ${ }^{1,2}$ Recently, a meta-analysis showed that chronic urticaria $(\mathrm{CU})$ is associated with higher risk of anxiety and 
depression $^{3}$ while a former systematic review reported a higher frequency of psychiatric disorders in CU, as anxiety, depression and somatoform disorders being the most common. ${ }^{4}$ Psychiatric disorders either can be a triggering factor, be the underlying cause or may appear as a consequence of $\mathrm{CU}$ during the course of the disease, the connection of these two entities has not been clarified yet. ${ }^{2}$

Omalizumab (OMA) is the only licensed treatment in antihistamine refractory CSU patients recommended by the latest EAACI/GA ${ }^{2}$ LEN/EDF/WAO guideline. ${ }^{5}$ Albeit showing high response rates in patients with CSU (OMA provided response rates of 52-90\% according to real-life studies and many clinical trials), OMA still fails to provide benefit in a proportion of patients. ${ }^{6,7}$ There have been efforts to define a biomarker to predict response to OMA, since this would be very beneficial for aiding decisions in clinical practice. It has recently been demonstrated that D-dimer and IgE levels may predict clinical response to OMA treatment in patients with CSU and some reported other potential biomarkers such as basophil FceRI expression, basophil histamine release assay, autologous serum skin test (ASST), and basophil activation test. ${ }^{8,11}$

Many studies and trials showed that OMA improves QoL significantly in patients with CSU. ${ }^{1,12,13}$ But few studies investigated whether it improves depressive symptoms or not and whether presence of comorbid psychiatric conditions affect treatment responses. In the current study, we aimed to investigate if depression scores change after treatment and if the presence of depressive symptoms have an impact on treatment responses or not.

\section{Methods}

Patients with CSU from Urticaria Excellence and Reference (UCARE) Center who received at least three injections (4-weekly) of OMA were included in the study. The study was approved by the Institutional Ethics Committee (number of IRB:48670771-514.10). Written informed consent were taken from all participants.

Patient characteristics including age, gender, disease duration, ASST result, concomitant angioedema, concurrent chronic inducible urticarias (CINDU) and daily life stress were reviewed retrospectively. Urticaria activity (UAS), Turkish Version of Chronic Urticaria QoL (CU-Q $\left.{ }^{2} \mathrm{oL}\right),{ }^{14}$ Beck-Depression Inventory (Beck-D) ${ }^{15}$ and Urticaria Control Test $(\mathrm{UCT})^{16}$ scores were evaluated before and after three injections of OMA (at week 12).

Beck-depression inventory (Beck-D) is a self-reported measure with 21 items each scored between 0 to 3 . It evaluates the cognitive, affective, and somatic symptoms of depression, experienced during the last 2 weeks and results were classified into 4 ranges: normal $<10$ points, mild depression-11 to 16 , moderate depression-17 to 20 , severe depression $>20$. Higher total scores indicate more severe depressive symptoms. ${ }^{15}$

Urticaria control test (UCT) is a 4-questioned tool, and each question has 5 answer options which are scored between 0 and 4 points. Total UCT score is the sum of all 4 individual item scores (0-16 points). A score of $\geq 12$ shows well-controlled urticaria and a score of $<12$ points indicates poor disease control. ${ }^{16}$

Urticaria activity score (UAS) is a self-completed daily diary combining the severity of pruritus and the number of wheals every day. Sum of the hives and pruritus scores is summed across 7 days to have UAS7 (0-42 points). Higher scores indicate higher activity.

Chronic urticaria quality of life questionnaire (CU$\mathbf{Q}^{2} \mathbf{o L}$ ) consists of 23 questions including six specific domains: itch, swelling, impact on life activities, sleep problems, looks and limits. It has a 2-week recall period and each question has 5 answer options (0 to 4 points), higher scores indicate worse QoL. ${ }^{14}$

Clinically meaningful changes in scores are also used to evaluate treatment responses. (17.5\% reduction in Beck-D score from baseline, 15 points decrease in $\mathrm{CU}-\mathrm{Q}^{2} \mathrm{OL}$ and a UCT score of 12 or more was defined as response to treatment. ${ }^{16}$ Patients with Beck-D scores $\geq 17$ were defined as having depressive symptoms.

Demographic data, patient reported outcome measures (PROMs) and treatment responses were compared between patients with depressive symptoms and without. Change in Beck-D-scores and other measures were evaluated before and after treatment.

\section{Statistical analysis}

All statistical analyses were performed using SPSS (IBM SPSS Statistics version 22). Numerical variables were reported as the mean \pm standard deviation, median, frequency and percentages. Differences in measured parameters between the patients with depressive symptoms and without or responders and non-responders were analyzed with Mann-Whitney U test such as, disease duration, scores of UAS7, UCT, Beck-D scores, and CU-Q ${ }^{2} \mathrm{oL}$. Difference in the age between the patients with depressive symptoms and without was analyzed with Student $\mathrm{T}$ test. The levels of significance values were for differences in UAS7, UCT, Beck-D scores, and CU-Q²oL following OMA treatment were calculated by Wilcoxon signed rank test. The comparison of the qualitative variables such as gender, ASST positivity, accompanying angioedema, and treatment responses of patients with depressive symptoms and without were analyzed with Pearson's chi-square test, Fisher's Exact test or Yates' Continuity Correction. Correlation between all variables was analyzed by Spearman's rho. Statistical significance was achieved at $p<0.05$ and $p<0.01$.

\section{Results \\ Patient characteristics}

A total of 49 patients with a mean age of $45.47 \pm 13.95$ years (range $18-77$, median $=44$ ) were enrolled in the study. Disease duration was $48.29 \pm 80.65$ months (3-480, median $=18)$ and $75,5 \%$ of the patients were women $(n=37)$. Thirty-three patients $(67.3 \%)$ had concomitant angioedema, 9 (18.4\%) had concurrent CINDUs and 19 (51.4\%) patients had positive ASST. Daily life stress has been reported by 40 patients $(81.6 \%)$ patients. 
Comparison of demographic data, patient reported outcome measures (PROMs) and treatment responses between patients with and without depressive symptoms

According to Beck-D scores at baseline, 20 (40.8\%) had depressive symptoms while 29 (59.2\%) did not have. When compared, there were no differences with respect to age, gender, disease duration, angioedema, ASST and UAS7 scores between patients with and without depressive symptoms ( $p$ $>0.05$ ) (Table 1). But UCT scores of patients with depressive symptoms were lower than the patients without depressive symptoms both at baseline and week 12 ( $p=0.04, p=0.006$, respectively). The $\mathrm{CU}-\mathrm{Q}^{2} \mathrm{OL}$ scores were higher at baseline and at week 12 in patients with depressive symptoms $(p=0.001$, $p=0.02$, respectively) (Table 1 ). And Beck-D scores at baseline correlated with CU-Q ${ }^{2} \mathrm{oL}$ scores $(r=0,398, p=0.005)$ while did not correlate with age, disease duration, UCT and UAS7 $(p>0.05)$. Changes in Beck-D scores and CU- ${ }^{2} \mathrm{oL}$ scores from baseline were significantly correlated at week 12 $(r=0.291, p=0.043)$. After treatment with OMA, UAS7, CU$\mathrm{Q}^{2} \mathrm{oL}$, Beck-D scores decreased and UCT-scores increased significantly from baseline ( $p<0.001$, for all) (Figure 1).

OMA-non-responders $(\mathrm{n}=12)$ had higher baseline and week 12 Beck-D-scores compared to responders $(\mathrm{n}=37)$ (median (interquartile range, IQR) $18.5(15.2-22)$ vs 12 (6-22.5); $p=0.031 ; 9(4.2-17.7)$ vs $4(0-7.5) ; p=0.01)$ (Figure 2$)$. The number of OMA non-responders were significantly higher among patients with depressive symptoms compared to patients without. ( $40 \%$ vs $13.8 \%$; $p=0.048$ ) (Table 1).

Beck-D-scores decreased significantly ( $17.5 \%$ reduction) from baseline in $39(79.6 \%)$ patients, and CU-QoL decreased significantly (15-points decrease) from baseline in $34(69.4 \%)$ and only $6(12.2 \%)$ patients scored as having depressive symptoms after treatment. Of these 6 patients only one was an OMA-responder.

Table 1. Comparison of demographic data, patient reported outcome measures (PROMs) and treatment responses between patients with depressive symptoms and without

\begin{tabular}{|c|c|c|c|c|}
\hline \multicolumn{2}{|c|}{$\begin{array}{l}\text { Patients with Chronic spontanous urticaria (CSU) } \\
\qquad n=49\end{array}$} & \multirow{2}{*}{$\begin{array}{l}\text { Patients without depressive } \\
\text { symptoms }(\mathrm{n}=\mathbf{2 9}, \mathbf{5 9 . 2 \% )} \\
477.90 \pm 14.11\end{array}$} & \multirow{2}{*}{$\begin{array}{c}\text { Patients with depressive } \\
\text { symptoms }(\mathrm{n}=\mathbf{2 0}, \mathbf{4 0 . 8} \%) \\
41.95 \pm 13.28\end{array}$} & \multirow{2}{*}{$\begin{array}{c}p \\
0.14^{\mathrm{a}}\end{array}$} \\
\hline Age (years) & mean \pm sd & & & \\
\hline Gender $(\mathrm{M} / \mathrm{F})$ & $(\mathrm{n}, \%)$ & $8(27.6 \%) / 21(72.4 \%)$ & $4(20 \%) / 16(80 \%)$ & $0.74^{\mathrm{b}}$ \\
\hline Disease duration (mo) & median (IQR) & $18(10$ to 54$)$ & $12(6.25$ to 45$)$ & $0.38^{c}$ \\
\hline Accompanying angioedema & $(\mathrm{n}, \%)$ & $20(69 \%)$ & $13(65 \%)$ & $0.77^{\mathrm{b}}$ \\
\hline ASST positivity & $(\mathrm{n}, \%)$ & $12(50 \%)$ & $7(53,8 \%)$ & $0.82^{\mathrm{b}}$ \\
\hline UCT at week 0 & median (IQR) & $5(2.5$ to 6.5$)$ & $2.5(1$ to 5$)$ & $0.04^{\mathrm{c}}$ \\
\hline UCT at week 12 & median (IQR) & $14(12$ to 16$)$ & $12(9$ to 13$)$ & $0.006^{c}$ \\
\hline Change in UCT (week 0-12) & median (IQR) & $9(6$ to 12.5$)$ & $9.5(7$ to 10.75$)$ & $0.83^{c}$ \\
\hline $\mathrm{UCT} \geq 12$ at week 12 & $(\mathrm{n}, \%)$ & $25(86.2 \%)$ & $12(60 \%)$ & $0.048^{\mathrm{b}}$ \\
\hline UAS7 at week 0 & median (IQR) & 25 (19.5 to 32.5$)$ & $28(19.5$ to 38$)$ & $0.34^{\mathrm{c}}$ \\
\hline UAS7 at week 12 & median (IQR) & $3(0$ to 14.5$)$ & $14(0.25$ to 24$)$ & $0.12^{c}$ \\
\hline Change in UAS7 (week 0-12) & median (IQR) & $-20(-26.5$ to -9$)$ & $-12(-25$ to -3.25$)$ & $0.36^{\mathrm{c}}$ \\
\hline $\mathrm{CU}-\mathrm{Q}^{2} \mathrm{oL}$ at week 0 & median (IQR) & $33(22$ to 41$)$ & $50(32.75$ to 60$)$ & $0.001^{\mathrm{c}}$ \\
\hline CU-Q²oL at week 12 & median (IQR) & $4(0.5$ to 10$)$ & $11(4.25$ to 30$)$ & $0.02^{\mathrm{c}}$ \\
\hline Change in CU-Q²oL (week 0-12) & median (IQR) & $-22(-36$ to -8.5$)$ & $-36(-53$ to -10.75$)$ & $0.16^{c}$ \\
\hline Beck-D score at week 0 & median (IQR) & $10(4.5$ to 12.5$)$ & $23(21$ to 27.75$)$ & $<0.001^{\mathrm{c}}$ \\
\hline Beck-D score at week 12 & median (IQR) & $4(0$ to 6.5$)$ & $8(2.25$ to 8$)$ & $0.007^{c}$ \\
\hline Change in Beck-D score (Week 0-12) & median (IQR) & $-5(-8.5$ to -1$)$ & $-16.5(-18$ to -9.5$)$ & $<0.001^{\mathrm{c}}$ \\
\hline
\end{tabular}

${ }^{a}$ student $t$ test; ${ }^{b}$ chi-square test; ${ }^{c}$ mann-whitney-U test

autolog serum skin test (ASST), Beck-depression inventory (Beck-D), chronic urticaria quality of life (CU-Q²oL), IQR, interquartile range (lower quartile - upper quartile), quality of life (QOL), sd, standard deviation, Urticaria activity score (UAS), Urticaria control test (UCT) 


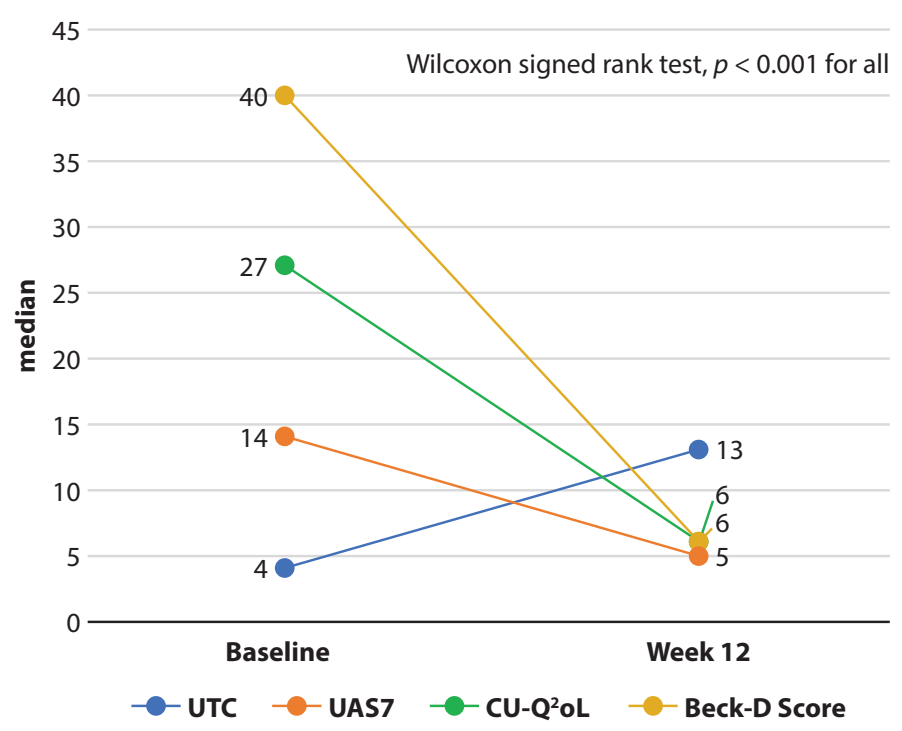

Figure 1. Change of patient related outcome measures (PROMs) after 3 months of treatment with omalizumab

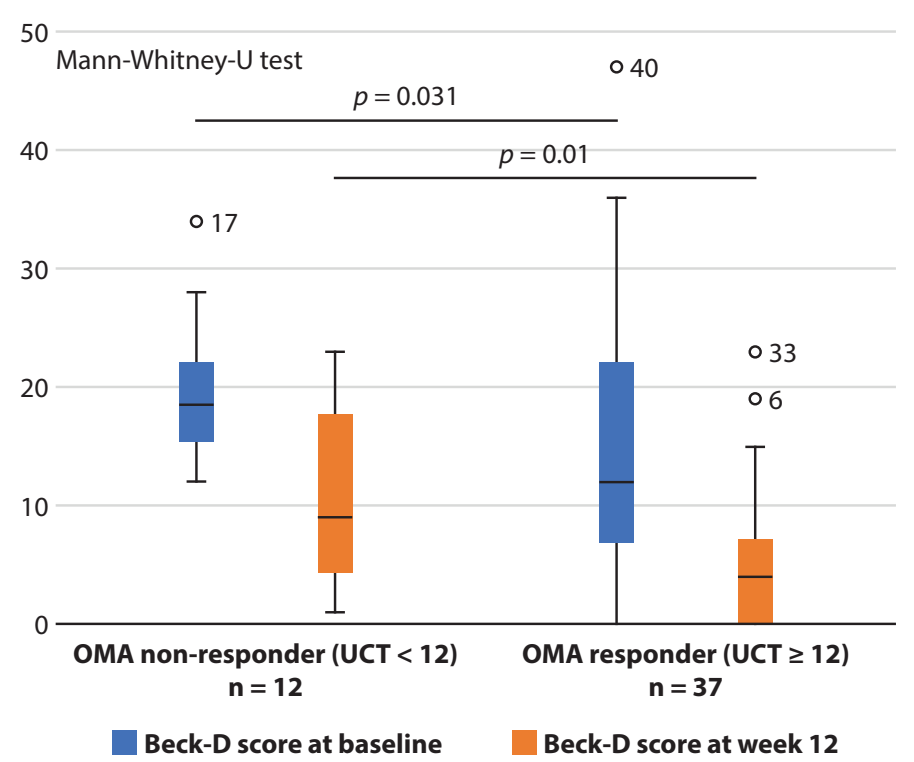

Figure 2. Comparison of Beck-D scores between omalizumab-responders and non-responders. Beck-D scores of omalizumab non-responder group were higher than the responder group at baseline and week 12

20 patients had depressive symptoms at baseline while only 6 patients were found to have depressive symptoms after 12 weeks of treatment supporting that, depression happens as a consequence of the disease which happens due to symptoms and their consequencies such as QoL impairment and sleep deprivation during the course of the disease. Recently, a study performed by Diluvio et al. ${ }^{25}$ revealed that CSU patients demonstrated markedly reduced physical health and psychological health subscale scores compared with controls and, after 6 months of treatment with OMA, 71\% of subjects who had obtained a borderline or abnormal score for depression obtained a normal score and $60 \%$ of subjects with a previous abnormal anxiety score achieved a normal score for anxiety. Similar to that study Patella $\mathrm{V}$ et al. ${ }^{26}$ found that psychological scores improved after 24 weeks of treatment with omalizumab. Our results are parallel to these studies; depressive symptoms substantially improved in $70 \%$ of our patients after 3 months of OMA treatment.

Although the exact mechanism of action of OMA in CSU is not known, the binding of free $\operatorname{IgE}$ and preventing the activation of mast cells and basophils are the main actions of OMA. ${ }^{27-28}$ The positive impact of OMA on depressive symptoms seen in our study may be due to the stabilization of mast cells and basophils so that they will not respond to stimulation via neuromediators such as substance $\mathrm{P}$, or it could be due to simply the disappearance of bothering urticaria symptoms. In the present study, OMA treatment, by decreasing the disease activity, improved the QoL of the patients significantly and led to improved Beck-D scores. Pooled analysis of ASTERIA I/II and GLACIAL showed that the mean change in dermatology life quality index (DLQI) scores were reported to be $30-50 \%$ greater in the OMA versus placebo group revealing that OMA effectively improved QoL. ${ }^{1} \mathrm{X}$-ACT study also supports the second theory and showed that angioedema-QoL 
and DLQI scores decreased rapidly following initiation of treatment, World Health Organization Well-being Index (WHO-5) of patients with CSU and angioedema increased above the depression threshold from week 4 and throughout OMA treatment but not placebo treatment and, approximately $80 \%$ of patients showed no signs of depression compared to approximately $40 \%$ in the placebo at week 28 of the treatment. This study also suggests that, psychological symptoms observed in CSU patients may be a consequence of the dermatological symptoms experienced. That study also showed that impaired WHO-5, correlated well with the markedly impaired QoL scores at baseline. ${ }^{12}$ Diluvio et al..$^{25}$ showed that there was a significant reduction in UAS7 $(p<0.001)$, anxiety scale, and DLQI $(p<0.01)$ after OMA therapy, but no significant decrease was observed in the depression scale although our study and X-ACT study ${ }^{12}$ revealed substantial improvements in depression scores. We found that QoL impairment was higher in patients with depressive symptoms. But the correlation was moderate between $\mathrm{CU}-\mathrm{Q}^{2} \mathrm{OL}$ and the Beck-D scores. Studies show a positive correlation between itch intensity and severity of depression and stress..$^{29,30}$

The current study showed that disease control was poorer in OMA treated CSU patients with depressive symptoms. Mental disorders might influence illness perception, behavior, preferences, satisfaction with medical procedures and adherence to treatment thereby impairing treatment results. ${ }^{17}$ In the present study, among 6 patients who were found to have depressive symptoms after treatment with OMA, five were OMA non-responders. The number of OMA non-responders were significantly higher ( $40 \%$ vs $13.8 \%)$ and UCT scores were lower among patients with depressive symptoms compared to patients without. Morever, OMA-non-responders had higher baseline and week 12 Beck-D-scores compared to responders. All these findings support the idea that depression might impair treatment responses, albeit the design of our study is not suited to make definite conclusions about this. Significant positive correlation between UAS7 and depression scores was shown recently ${ }^{31}$ and the severity of psychiatric disease was also found to correlate with QoL impairment. ${ }^{21,32}$ Increased levels of stress may evoke or aggravate CSU symptoms ${ }^{33}$ and in a recent study, stress levels were found to be linked to disease activity. ${ }^{34}$ But unfortunately, there is not enough evidence on the efficacy of psychiatric interventions and treatments on CSU patients, but few reports showed that antidepressant or anti-anxiety drugs can improve the symptoms of $\mathrm{CU}$ which may support depression as a causative or excerbating factor rather than a consequence of the disease. ${ }^{2,35}$

Therefore, we believe that the presence of psychiatric comorbidities should be managed accordingly if they coexist with CSU since depression may exacerbate the symptoms and impairs the QoL by changing the perception of the patients and may impair response to treatment.

Limitations of this study are first; it would be ideal to refer the patients for psychiatric evaluation but since we could not do this, only having Beck-D scores resulted in defining of the patients as having depressive symptoms instead of depression.

\section{Conclusion}

Depression and quality of life impairment is common among CSU patients. Presence of depression is associated with lower quality of life and poorer disease control. Treatment with omalizumab improves disease activity, quality of life and depression scores. Depression seems to occur as a consequence of CSU rather than being a cause of it. Presence of depression might impair treatment responses. The study emphasizes the necessity of psychological evaluation and a comprehensive, multidisciplinary approach including psychiatrists in the management of this chronic, debilitating disease.

\section{Funding Sources}

This article has no funding source. This research did not receive any specific grant from funding agencies in the public, commercial, or not-for-profit sectors.

\section{Conflict of Interest Disclosure}

Dr. Emek Kocaturk reports advisory board fees from Novartis, and has served as a medical advisor for Bayer, Sanofi and Menarini. The other authors have no conflict of interest to declare.

\section{Ethics approval}

The study was approved by the Institutional Ethics Committee of Okmeydani Training and Research Hospital (number of IRB: 48670771-514.10).

\section{Author contributions}

- EK, PKC, PE, END contributed to the conception and design of the study, editing the figures, and preparation, drafted the article and critical revision of the manuscript.

- PKC contributed to analysis of the study.

- EK, PKC, PE, END revised and approved the final version of the manuscript.

- EK and PE, PKC contributed to the acquisition and interpretation of the data and revised critically.

- The manuscript has been read and approved by all the authors, that the requirements for authorship as stated earlier in this document have been met, and that each author believes that the manuscript represents honest work.

\section{References}

1. Maurer M, Sofen H, Ortiz B, F Kianifard, S Gabriel, JA Bernstein. Positive impact of omalizumab on angioedema and quality of life in patients with refractory chronic idiopathic/spontaneous urticaria: analyses according to the presence or absence of angioedema. J Eur Acad Dermatology Venereol. 2017;31:1056-63.

2. Konstantinou GN, Konstantinou GN. Psychiatric comorbidity in chronic urticaria patients: A systematic review and meta-analysis. Clin Transl Allergy. 2019;23:42.

3. Huang Y, Xiao Y, Zhang X, Li J, Chen X, Shen M. A Meta-Analysis of Observational Studies on the Association of Chronic Urticaria With Symptoms of Depression and Anxiety. Front Med. 2020;27:39.

4. Ben-Shoshan M, Blinderman I, Raz A. Psychosocial factors and chronic spontaneous urticaria: a systematic review. Allergy. 2013;68:131-41.

5. Zuberbier T, Aberer W, Asero R, Abdul Latiff AH, Baker D, Ballmer-Weber B, et al. The EAACI/GA2LEN/EDF/WAO Guideline for the Definition, Classification, Diagnosis and Management of Urticaria. Allergy. 2018;73:1393-414. 
6. Kocatürk E, Zuberbier T. New biologics in the treatment of urticaria. Curr Opin Allergy Clin Immunol. 2018;18:425-31.

7. Tharp MD, Bernstein JA, Kavati A, Ortiz B, MacDonald K, Denhaerynck $\mathrm{K}$, et al. Benefits and Harms of Omalizumab Treatment in Adolescent and Adult Patients with Chronic Idiopathic (Spontaneous) Urticaria: A Meta-analysis of "real-world" Evidence. JAMA Dermatology. 2019;155: 29-38.

8. Kocatürk E, Kızıltaç K, Tabi L. Omalizumab in chronic urticaria: A comprehensive review. Turkderm-Turk Arch Dermatol Venereology. 2018;52:112-19.

9. Marzano AV, Genovese G, Casazza G, Fierro MT, Dapavo P, Crimi N, et al. Predictors of response to omalizumab and relapse in chronic spontaneous urticaria: a study of 470 patients. J Eur Acad Dermatology Venereol. 2019; 33:918-24.

10. Ghazanfar MN, Thomsen SF. Predicting Response to Omalizumab in Chronic Urticaria Based on Biomarkers. EMJ Dermatol. 2018;6:124-30.

11. Weller K, Ohanyan T, Hawro T, Ellrich A, Sussman G, Koplowitz J, et al. Total IgE levels are linked to the response of chronic spontaneous urticaria patients to omalizumab. Allergy. 2018;73:2406-8.

12. Staubach P, Metz M, Chapman-Rothe N, Sieder C, Brautigam M, Maurer $\mathrm{M}$, et al. Omalizumab rapidly improves angioedema-related quality of life in adult patients with chronic spontaneous urticaria: X-ACT study data. Allergy. 2018;73:576-84.

13. Salman A, Demir G, Bekiroglu N. The impact of omalizumab on quality of life and its predictors in patients with chronic spontaneous urticaria: Real-life data. Dermatol Ther. 2019;32:e12975.

14. Kocatürk E, Weller K, Martus P, Aktaș S, Kavala M, Sarıgül S, et al. Turkish version of the chronic urticaria quality of life questionnaire: cultural adaptation, assessment of reliability and validity. Acta Derm Venereol. 2012;92:419-25.

15. Tuğlu C, Türe M, Dağdeviren N, Aktürk Z. The reliability and validity analysis of the Turkish version of BECK depression inventory for primary care. Turkish Journal of Family Practice. 2005;9:117-22.

16. Weller K, Groffik A, Church MK, Hawro T, Krause K, Metz M, et al. Development and validation of the Urticaria Control Test: A patient-reported outcome instrument for assessing urticaria control. J Allergy Clin Immunol. 2014;133:1365-72.

17. Staubach P, Dechene M, Metz M, Magerl M, Siebenhaar F, Weller K, et al. High prevalence of mental disorders and emotional distress in patients with chronic spontaneous urticaria. Acta Derm Venereol. 2011;91:557-61.

18. Mehta V, Malhotra SK. Psychiatric Evaluation of Patients with Psoriasis Vulgaris and Chronic Urticaria. Ger J Psychiatry. 2007;10:104-10.

19. Sorour F, Abdelmoaty A, Bahary MH, Birqdar BE. Psychiatric disorders associated with some chronic dermatologic diseases among a group of Egyptian dermatology outpatient clinic attendants. J Egypt Women's Dermatologic Soc. 2017;14:31-6.
20. Bashir K, Dar NR, Rao SU. Depression in adult dermatology outpatients. J Coll Physicians Surg Pak. 2010;20:811-13.

21. Staubach P, Eckhardt-Henn A, Dechene M, Vonend A, Metz M, Magerl $\mathrm{M}$, et al. Quality of life in patients with chronic urticaria is differentially impaired and determined by psychiatric comorbidity. Br J Dermatol. 2006;154:294-8.

22. Georgin-Lavialle S, Moura DS, Salvador A, Chauvett Gelinier JC, Launay JM, Damaj G, et al. Mast cells' involvement in inflammation pathways linked to depression: Evidence in mastocytosis. Mol Psychiatry. 2016;21: 1511-6.

23. Conti P, Caraffa A, Ronconi G, Conti CM, Kritas SK, Mastrangelo F, et al. Impact of mast cells in depression disorder: inhibitory effect of IL-37 (new frontiers). Immunol Res. 2018;66:323-31.

24. Nautiyal KM, Ribeiro AC, Pfaff DW, Silver R. Brain mast cells link the immune system to anxiety-like behavior. Proc Natl Acad Sci USA. 2008; 105:18053-7.

25. Diluvio L, Piccolo A, Marasco F, Vollono L, Lanna C, Chiaramonte B et al. Improving of psychological status and inflammatory biomarkers during omalizumab for chronic spontaneous urticaria. Future Sci OA. 2020;6(9): FSO618.

26. Patella V, Roberta Z, Florio G, Palmieri M, Palmieri S, Brancaccio R. Omalizumab improves perceived stress, anxiety and depression in chronic spontaneous urticaria. J Allergy Clin Immunol Pract. 2020;20:31247-2.

27. Bracken SJ, Abraham S, MacLeod AS. Autoimmune theories of chronic spontaneous urticaria. Front Immunol. 2019;29:627.

28. Chang TW, Chen C, Lin CJ, Metz M, Church MK, Maurer M. The potential pharmacologic mechanisms of omalizumab in patients with chronic spontaneous urticaria. J Allergy Clin Immunol. 2015;135:337-42.

29. Gupta MA, Gupta AK, Schork NJ, Ellis CN. Depression modulates pruritus perception: a study of pruritus in psoriasis, atopic dermatitis, and chronic idiopathic urticaria. Psychosom Med. 1994;56:36-40.

30. Ograczyk-Piotrowska A, Gerlicz-Kowalczuk Z, Pietrzak A. Stress, itch and quality of life in chronic urticaria females. Postepy Dermatol Alergol. 2018;35:156-60.

31. Tat TS. Higher levels of depression and anxiety in patients with chronic urticaria. Med Sci Monit. 2019;4:115-20.

32. Engin B, Uguz F, Yilmaz E, Ozdemir M, Mevlitoglu I. The levels of depression, anxiety and quality of life in patients with chronic idiopathic urticaria. J Eur Acad Dermatology Venereol. 2008;22:36-40.

33. Weller K, Koti I, Makris M, Maurer M. Anxiety and depression seem less common in patients with autoreactive chronic spontaneous urticaria. Clin Exp Dermatol. 2013;38:870-3.

34. Schut C, Magerl M, Hawro T, Kupfer J, Rose M, Gieler U, et al. Disease activity and stress are linked in a subpopulation of chronic spontaneous urticaria patients. Allergy. 2020;75:224-6.

35. Yasharpour MR, Randhawa I. Antidepressants in chronic idiopathic urticaria. Allergy Asthma Proc. 2011;32:419-24. 